\title{
ON OPTIMAL ALLOCATION OF A CONTINUOUS RESOURCE USING AN ITERATIVE APPROACH AND TOTAL POSITIVITY
}

\author{
JAY BARTROFF ${ }^{* * *}$ AND \\ LARRY GOLDSTEIN, ${ }^{*}$ University of Southern California \\ YOSEF RINOTT ${ }^{* * *}$ AND \\ ESTER SAMUEL-CAHN, ${ }^{* * *}$ The Hebrew University of Jerusalem
}

\begin{abstract}
We study a class of optimal allocation problems, including the well-known bomber problem, with the following common probabilistic structure. An aircraft equipped with an amount $x$ of ammunition is intercepted by enemy airplanes arriving according to a homogeneous Poisson process over a fixed time duration $t$. Upon encountering an enemy, the aircraft has the choice of spending any amount $0 \leq y \leq x$ of its ammunition, resulting in the aircraft's survival with probability equal to some known increasing function of $y$. Two different goals have been considered in the literature concerning the optimal amount $K(x, t)$ of ammunition spent: (i) maximizing the probability of surviving for time $t$, which is the so-called bomber problem; and (ii) maximizing the number of enemy airplanes shot down during time $t$, which we call the fighter problem. Several authors have attempted to settle the following conjectures about the monotonicity of $K(x, t)$ : (A) $K(x, t)$ is decreasing in $t$; (B) $K(x, t)$ is increasing in $x$; and (C) the amount $x-K(x, t)$ held back is increasing in $x$. Conjectures (A) and (C) have been shown for the bomber problem with discrete ammunition, while (B) is still an open question. In this paper we consider both time and ammunition to be continuous, and, for the bomber problem, we prove $(\mathrm{A})$ and $(\mathrm{C})$, while, for the fighter problem, we prove (A) and $(\mathrm{C})$ for one special case and (B) and (C) for another. These proofs involve showing that the optimal survival probability and optimal number shot down are totally positive of order $2\left(\mathrm{TP}_{2}\right)$ in the bomber and fighter problems, respectively. The $\mathrm{TP}_{2}$ property is shown by constructing convergent sequences of approximating functions through an iterative operation which preserves $\mathrm{TP}_{2}$ and other properties.
\end{abstract}

Keywords: Bomber problem; log-concave; optimal allocation; sequential optimization; total positivity

2010 Mathematics Subject Classification: Primary 60G40

Secondary 62L05; 91A60

\section{Introduction}

Throughout this paper, we write decreasing for nonincreasing and increasing for nondecreasing. When we use strict monotonicity, we will say so explicitly. The allocation problem

Received 11 January 2010; revision received 26 April 2010.

* Postal address: Department of Mathematics, University of Southern California, 1042 West 36th Place, Los Angeles, CA 90089-1113, USA.

** Email address: bartroff@usc.edu

*** Postal address: Department of Statistics and Center for the Study of Rationality, The Hebrew University of Jerusalem, Jerusalem, 91905, Israel. 
discussed in this paper can be described in terms of the following example: an aircraft, which is at flying time $t$ away from its final destination, is equipped with an amount $x$ of ammunition. It is confronted by enemy airplanes whose appearance is driven by a time-homogenous Poisson process with known intensity. By adjusting the units of time, we assume without loss of generality that the intensity is 1 . It is assumed that the encounters are instantaneous, i.e. that their duration time is 0 . The question of interest is: how much of the presently available ammunition should be spent when confronted by an enemy while in 'state' $(x, t)$ ? We consider two problems, the bomber problem and the fighter problem, with this common probabilistic setup. For the bomber problem, the goal is to maximize the probability $P(x, t)$ of reaching its destination. For the fighter problem, the goal is to maximize the expected number, $N(x, t)$, of enemy airplanes shot down. In both problems there is given an increasing function $a(y)$, which in the bomber problem is the probability that the bomber survives an enemy encounter in which it expends $0 \leq y \leq x$ units of its ammunition, while in the fighter problem $a(y)$ is the probability that the fighter destroys an enemy when it expends $y$ units of its ammunition. Although our results below are proved when $a(y)$ is general, the canonical example considered in the literature for $a(y)$ in the bomber problem is

$$
1-(1-u) \mathrm{e}^{-y}
$$

for some fixed $u \in[0,1]$, where $1-\mathrm{e}^{-y}$ is the probability that the bomber's $y$ units of ammunition destroy the enemy and, failing to do so, $1-u$ is the probability that the enemy's counterattack succeeds.

For the fighter, where $a(y)$ is the probability of destroying the enemy, the probability that the fighter survives an encounter is $a(y)+u(1-a(y))$, where $a(y)$ is the probability that the fighter's $y$ units of ammunition destroy the enemy and, failing to do so, $u$ is the probability that the enemy's counterattack fails. When taking the canonical choice $a(y)=1-\mathrm{e}^{-y}$, the fighter's survival is given by (1.1).

While the optimal ammunition spending strategies $K(x, t)$ may differ for the bomber and fighter problems, it is perhaps intuitively obvious that the following three monotonicity properties, first posed in Klinger and Brown (1968) for the bomber problem, should hold for both:

(A) $K(x, t)$ is decreasing in $t$;

(B) $K(x, t)$ is increasing in $x$;

(C) $x-K(x, t)$ is increasing in $x$.

Roughly speaking, (A) states that the closer one is to the destination, the more one should spend, (B) states that the more ammunition one has, the more one should spend, and (C) states that the more ammunition one has, the more one should retain for possible future encounters; replacing 'more' by 'not less' makes these characterizations precise. Although we write $K(x, t)$ for the two different goals, no confusion should arise, as it will always be clear which of the problems we are discussing, or that we are discussing them simultaneously. An optimal policy may not be unique. If it is not unique, $K(x, t)$ will always denote the minimal optimal amount of ammunition needed.

Although the paper is written in plane English, the settings above serve only as illustrations of the general method which can be applied to a wide class of problems where some limited resource needs to be optimally allocated over time or space to maximize the probability of a system's survival, which clearly also have more peaceful applications. 
Of the two problems, the bomber problem has received most attention. For discrete ammunition, such as missiles, it was first treated in Klinger and Brown (1968), who proved that (B) implies (A). A clearer picture emerges in Samuel (1970), who proved (A) without assuming (B). Samuel's (1970) formula (3.9) also proves (C). Conjecture (B) has remained elusive, and in fact remains in doubt. Simons and Yao (1990) argued that when both time and ammunition are continuous, then (B) is equivalent to the $\log$ concavity of $P(x, t)$ in $x$ for each fixed $t$. They also showed that when both time and ammunition are discrete, there exist parameter values for which log concavity fails. However, they were unable to supply an example where (B) fails.

Regarding the fighter problem, we are unaware of this variation on the bomber problem being discussed in a finite time $t$ horizon setting. For an infinite time horizon, an optimal policy can be written simply as $K(x)$. Weber (1985) showed that the monotonicity of $K(x)$ fails to hold for discrete ammunition when $a$ is given by (1.1) with $u=0$, and Shepp et al. (1991) showed that monotonicty fails for continuous ammunition. In a setting where both ammunition and time are discrete, the duration $T$ is an unknown exponential random variable with known mean, and geometric arrivals of the enemy. Shepp et al. (1991) also showed that there exist parameter values for which (B) fails. Though this variant is on a finite time interval of length $T$, the optimal policy does not depend on $t$ and so resembles an infinite time horizon problem. On the other hand, Bartroff et al. (2010) showed that, for the bomber problem with continuous time and ammunition, there exists a certain region of the $(x, t)$-space over which $K(x, t)=x$, a monotone function of $x$, and, hence, (B) holds in this region.

In the present paper we consider the 'doubly continuous' setting where ammunition and time are continuous variables. Given an increasing function $a(y)$ taking values in $[0,1]$, the dynamic programming equation for the bomber problem giving the optimal survival probability $P(x, t)$ is

$$
P(x, t)=\int_{0}^{t} \sup _{0 \leq y \leq x} a(y) P(x-y, t-s) \mathrm{e}^{-s} \mathrm{~d} s+\mathrm{e}^{-t} \quad \text { for }(x, t) \in \mathbb{R}^{+} \times \mathbb{R}^{+},
$$

where $\mathbb{R}^{+}=[0, \infty)$. The explanation is as follows. There are no encounters in a time interval of length $t$ with probability $\mathrm{e}^{-t}$, yielding the final term. Otherwise, an encounter occurs at time $s \in[0, t]$ with density $\mathrm{e}^{-s}$, at which time the amount $y \in[0, x]$ will be chosen to maximize the probability $a(y)$ of surviving the current encounter times the probability of future survival under an optimal policy. A simple change of variables yields

$$
P(x, t)=\int_{0}^{t} \sup _{0 \leq y \leq x} a(y) P(x-y, s) \mathrm{e}^{-(t-s)} \mathrm{d} s+\mathrm{e}^{-t} \quad \text { for }(x, t) \in \mathbb{R}^{+} \times \mathbb{R}^{+} .
$$

Given an increasing function $a(y)$ taking values in $[0,1]$ and a fixed value $u \in[0,1]$, the dynamic programming equation for the fighter problem giving the optimal expected number $N(x, t)$ of enemies shot down is

$$
N(x, t)=\int_{0}^{t} \sup _{0 \leq y \leq x}\{a(y)+[a(y)+u(1-a(y))] N(x-y, s)\} \mathrm{e}^{-(t-s)} \mathrm{d} s
$$

for $(x, t) \in \mathbb{R}^{+} \times \mathbb{R}^{+}$, which can be interpreted as follows. Encounters occur with density $\mathrm{e}^{-s}$, and expending $y$ units of ammunition the fighter gains a single hit if he destroys the enemy, which happens with probability $a(y)$. The fighter will gain an expected additional $N(x-y, t-s)$ future hits if he destroys the enemy or if the enemy's counterattack fails, which happens with probability $u$. A change of variables then yields (1.3).

By putting both the bomber and the fighter in a common general framework, we show that solutions to (1.2) and (1.3) exist and are unique and continuous. In particular, the supremum is 
attained in both (1.2) and (1.3), and, hence, sup can be replaced by max in each. The optimal policy $K(x, t)$ is then defined as the minimal value for which the max in the integrands in (1.2) and (1.3) is attained.

Recall that a nonnegative function $Q(x, t)$ is totally positive of order 2 , written $\operatorname{TP}_{2}(x, t)$ or simply $\mathrm{TP}_{2}$, if

$$
Q\left(x^{\prime}, t^{\prime}\right) Q(x, t) \geq Q\left(x^{\prime}, t\right) Q\left(x, t^{\prime}\right) \quad \text { whenever } x<x^{\prime} \text { and } t<t^{\prime},
$$

and strictly $\mathrm{TP}_{2}$ if ' $\geq$ ' in (1.4) can be replaced by ' $>$ '. (See Karlin (1968, p. 46) and the references therein.) To prove conjecture (A) for the bomber problem, we establish the $\mathrm{TP}_{2}$ property of $P(x, t)$ in Section 3. Recall also that a log-concave function is a function whose logarithm is concave.

Theorem 1.1. Let $a(y)$ be a uniformly continuous log-concave function. Then a unique bounded solution $P(x, t)$ to (1.2) exists, satisfies $P(x, t) \in[0,1]$, is continuous, and $T P_{2}$.

Theorem 1.2. If a $(y)$ is a uniformly continuous log-concave function, then conjecture (A) holds for the doubly continuous bomber problem.

Simons and Yao (1990) claimed that conjecture (C) could be shown to hold for continuous $x$ and $t$ by arguments similar to those they provided for the case where both $x$ and $t$ are discrete; in Section 3 we provide a rigorous proof of (C) for the doubly continuous case. The discrete $x$ analogues to Theorems 1.1 and 1.2 were proved in Samuel (1970).

Regarding the fighter in general, we have the following result.

Theorem 1.3. If $a(y)$ is uniformly continuous then, for any $u \in[0,1]$, a bounded solution $N(x, t)$ to (1.3) exists, is unique, satisfies $N(x, t) \in[0, t]$, and is continuous.

For the fighter problem, we do not resolve the question of whether the general solution to $(1.3)$ is $\mathrm{TP}_{2}$, but instead examine two special cases in more detail.

Case (i). The frail fighter: the $u=0$ case. Once the frail fighter fails to shoot down an enemy, he himself is shot down with probability 1. Equation (1.3) then simplifies to

$$
N(x, t)=\int_{0}^{t} \sup _{0 \leq y \leq x} a(y)[1+N(x-y, s)] \mathrm{e}^{-(t-s)} \mathrm{d} s \quad \text { for }(x, t) \in \mathbb{R}^{+} \times \mathbb{R}^{+} .
$$

Theorem 1.4. If a $(y)$ is a uniformly continuous log-concave function then the solution $N(x, t)$ is $T P_{2}$, and conjecture (A) holds for the frail fighter.

The frail fighter is discussed in Section 4, where conjecture (C) is also established.

Case (ii). The invincible fighter: the $u=1$ case. As the name suggests, the invincible fighter cannot be shot down. Equation (1.3) simplifies to

$$
N(x, t)=\int_{0}^{t} \sup _{0 \leq y \leq x}[a(y)+N(x-y, s)] \mathrm{e}^{-(t-s)} \mathrm{d} s \quad \text { for }(x, t) \in \mathbb{R}^{+} \times \mathbb{R}^{+} .
$$

Theorem 1.5. If a $(y)$ is a uniformly continuous concave function then conjecture $(B)$ holds for the invincible fighter.

The invincible fighter is discussed in Section 5, where we also show that $(\mathrm{C})$ holds and that the maximum $y$ determining $K(x, t)$ is uniquely attained. It should be noted that we have not managed to show that (A) and (B) hold simultaneously for any of the problems we consider. 
While in the discrete ammunition case it is possible to demonstrate claims using induction on the number of available units (see, e.g. Simons and Yao (1990)), here we must take a different approach. In Section 2 we prove the existence and uniqueness of solutions to equations which have the form of (1.2) or (1.3) by following the outline suggested by Weber (see Simons and Yao (1990, p. 431)), and we construct a sequence of functions through an iteration which converges exponentially fast to the solution.

We illustrate our approach using the bomber problem; similar remarks apply to the fighter problem. First, consider a slightly transformed version of (1.2). For ease of notation, for functions $a(\cdot)$ and $Q(\cdot, \cdot)$, let

$$
(a \star Q)(x, s)=\sup _{0 \leq y \leq x} a(y) Q(x-y, s)
$$

denote the sup-convolution of $a(\cdot)$ and $Q(\cdot, s)$. Letting

$$
\bar{P}(x, t)=\mathrm{e}^{t} P(x, t),
$$

substitution into (1.2) results in the integral equation

$$
\bar{P}(x, t)=\int_{0}^{t}(a \star \bar{P})(x, s) \mathrm{d} s+1 \quad \text { for }(x, t) \in \mathbb{R}^{+} \times \mathbb{R}^{+} .
$$

Clearly, there exists a unique, continuous solution $\bar{P}$ to (1.7) satisfying $0 \leq \bar{P}(x, t) \leq \mathrm{e}^{t}$ if and only if there exists a unique, continuous solution $P$ to (1.2) satisfying $0 \leq P(x, t) \leq 1$; Theorem 2.1, below, proves the former and provides an iteration method to approximate the solution. Theorem 3.1, below, provides further properties of the solution.

To prove the claims of Theorem 3.1, consider the sequence of functions $\left\{\bar{P}_{m}\right\}_{m \geq 0}$ generated with some initial $\bar{P}_{0}(x, t)$ by the recursion

$$
\bar{P}_{m+1}(x, t)=\int_{0}^{t}\left(a \star \bar{P}_{m}\right)(x, s) \mathrm{d} s+1 \quad \text { for } m \geq 0 .
$$

In Section 2, by showing that the operation which gives $\bar{P}_{m+1}$ from $\bar{P}_{m}$ is a type of contraction, we are able to prove the existence and uniqueness of the solution to (1.8) by applying the methods of the contraction mapping theorem. In Section 3 we prove that various operations preserve the $\mathrm{TP}_{2}$ and other properties, allowing us to prove inductively that $\bar{P}_{m}$ possesses a particular property for all $m \geq 0$, which may then be inherited by $\bar{P}$. We remark in Section 6 why this same approach appears to fail to give conjecture (B). Nevertheless, the sequence of approximate solutions, which are guaranteed to converge in the supremum norm at an exponential rate by Theorem 2.1, may yet have uses in addition to that of proving conjecture (A).

\section{Existence, uniqueness, and approximating sequences}

We will put our models into the following common framework. Given a function $a: \mathbb{R}^{+} \rightarrow$ $\mathbb{R}^{+}$, we consider an operation ' $\otimes$ ' that, for a given function $Q: \mathbb{R}^{+} \times \mathbb{R}^{+} \rightarrow \mathbb{R}^{+}$, returns a function $a \otimes Q: \mathbb{R}^{+} \times \mathbb{R}^{+} \rightarrow \mathbb{R}^{+}$. Although our results in this section are more general, we will be interested in the specific cases where $(a \otimes Q)(x, t)$ is one of the following:

(Bo) $\sup _{0 \leq y \leq x} a(y) Q(x-y, t)$;

$\left(\mathrm{F}_{0}\right) \sup _{0 \leq y \leq x} a(y)\left[\mathrm{e}^{t}+Q(x-y, t)\right]$; 
$\left(\mathrm{F}_{1}\right) \sup _{0 \leq y \leq x}\left[a(y) \mathrm{e}^{t}+Q(x-y, t)\right]$;

$\left(\mathrm{F}_{u}\right) \sup _{0 \leq y \leq x}\left\{a(y) \mathrm{e}^{t}+[a(y)+u(1-a(y))] Q(x-y, t)\right\}$.

Case (Bo) corresponds to the bomber problem (1.7), case $\left(\mathrm{F}_{u}\right)$ corresponds to the general fighter equation (1.3) after a rescaling by $\mathrm{e}^{t}$ as in (1.6), case $\left(\mathrm{F}_{0}\right)$ corresponds to the $u=0$ case of $\left(\mathrm{F}_{u}\right)$, the frail fighter, and case $\left(\mathrm{F}_{1}\right)$ corresponds to the $u=1$ case of $\left(\mathrm{F}_{u}\right)$, the invincible fighter.

Given $a$ and ' $\otimes$ ', we will consider operators mapping the collection of functions $Q: \mathbb{R}^{+} \times$ $\mathbb{R}^{+} \rightarrow \mathbb{R}^{+}$to itself. For the fighter problem, these operators will be of the form

$$
\mathcal{g}(x, t, Q)=\int_{0}^{t}(a \otimes Q)(x, s) \mathrm{d} s,
$$

and, for the bomber problem,

$$
\mathcal{F}(x, t, Q)=\int_{0}^{t}(a \otimes Q)(x, s) \mathrm{d} s \quad \text { and } \quad \mathcal{g}(x, t, Q)=\mathcal{F}(x, t, Q)+1 .
$$

For simplicity, in what follows we write $g(Q)$ in place of $g(x, t, Q)$. For a given function $Q_{0}(x, t)$ on $\mathbb{R}^{+} \times \mathbb{R}^{+}$, we study the sequence of iterates of $g$ given by

$$
Q_{m+1}(x, t)=g\left(x, t, Q_{m}\right) \quad \text { for } m \geq 0 .
$$

The main result of this section, given in Theorems 2.1 and 2.2, below, proves the existence and uniqueness of a solution $\bar{Q}$ to the equation

$$
\bar{Q}(x, t)=g(x, t, \bar{Q}),
$$

and the exponential rate of convergence of the sequence $Q_{m}$ to $\bar{Q}$ when $Q_{0}$ is continuous and $a$ and $\otimes$ satisfy certain continuity-type properties. These conditions make $g$ into a type of contraction, allowing us to apply the methods of the contraction mapping theorem (see, for instance, Luenberger (1969, Theorem 1, p. 272)) to prove that sequences of functions generated by the iterates of $g$ on some initial function converge to a unique fixed point of $g$.

For a function $Q$ bounded over all compact domains $D \subset \mathbb{R}^{+} \times \mathbb{R}^{+}$, let

$$
\|Q\|_{D}=\sup _{(y, s) \in D}|Q(y, s)|
$$

For any two functions $Q$ and $R$ defined on $D \subset \mathbb{R}^{+} \times \mathbb{R}^{+}$and $\delta \in[0, \infty)$, let

$$
\mathrm{d}_{D, \delta}^{1}(Q, R)=\sup _{\left\{(x, t),\left(x^{\prime}, t\right) \in D:\left|x-x^{\prime}\right| \leq \delta\right\}}\left|Q(x, t)-R\left(x^{\prime}, t\right)\right|
$$

and

$$
\mathrm{d}_{D, \delta}(Q, R)=\sup _{\left\{(x, t),\left(x^{\prime}, t^{\prime}\right) \in D:\left|x-x^{\prime}\right| \leq \delta,\left|t-t^{\prime}\right| \leq \delta\right\}}\left|Q(x, t)-R\left(x^{\prime}, t^{\prime}\right)\right| .
$$

The functions $\mathrm{d}_{D, \delta}^{1}$ and $\mathrm{d}_{D, \delta}$ satisfy all the properties of a metric except that they are not necessarily 0 when $Q=R$. 
Definition 2.1. Given a function $a(y)$, the operation ' $\otimes$ ' is of $\kappa$-contraction type for some $\kappa \geq 0$ if, for all compact domains $D=[0, X] \times[0, T]$, there exist $\beta \geq 0$ and $\eta(\delta) \geq 0$, defined for $\delta \geq 0$ and satisfying

$$
\eta(0)=0 \quad \text { and } \quad \lim _{\delta \downarrow 0} \eta(\delta)=0,
$$

such that, for any functions $Q$ and $R$ on $\mathbb{R}^{+} \times \mathbb{R}^{+}$,

$$
\mathrm{d}_{D, \delta}^{1}((a \otimes Q),(a \otimes R)) \leq \kappa \mathrm{d}_{D, \delta}^{1}(Q, R)+\eta(\delta)\left(\|Q\|_{D} \vee\|R\|_{D}+\beta\right)
$$

for all $\delta \in[0, \infty)$, where $b \vee c$ is the maximum of $b$ and $c$. The operation ' $\otimes$ ' is of contraction type if it is of $\kappa$-contraction type for some $\kappa \geq 0$.

Lemma 2.1. Let $g$ be given by (2.1) or (2.2) for ' $\otimes$ ' of $\kappa$-contraction type. Let $X \geq 0$, $T^{\prime} \geq T \geq 0$ be arbitrary, and set $D^{\prime}=[0, X] \times\left[0, T^{\prime}\right]$ and $D=[0, X] \times[0, T]$. If $Q$ and $R$ are any functions defined on $D^{\prime}$, then

$$
\|\mathscr{g}(Q)-\mathcal{G}(R)\|_{D^{\prime}} \leq \kappa T\|Q-R\|_{D}+\kappa\left(T^{\prime}-T\right)\|Q-R\|_{D^{\prime}}
$$

and when $a(y)$ is bounded, for all $\delta \geq 0$, the inequality

$$
\mathrm{d}_{D, \delta}(\mathscr{g}(Q), \mathscr{g}(R)) \leq \kappa T \mathrm{~d}_{D, \delta}^{1}(Q, R)+\omega(\delta)\left(\|Q\|_{D} \vee\|R\|_{D}+\gamma\right)
$$

holds for some $\gamma \geq 0$ and $\omega(\delta) \geq 0$ satisfying $\omega(0)=0$ and $\lim _{\delta \downarrow 0} \omega(\delta)=0$.

Proof. Clearly, it suffices to prove the claim for $g$ of the form (2.1). To obtain (2.6), let $(x, t) \in D^{\prime}$. Applying (2.5) with $\delta=0$, and noting that $\mathrm{d}_{D, 0}^{1}(Q, R)=\|Q-R\|_{D}$, we have

$$
\begin{aligned}
\mid \mathcal{G}(x, & t, Q)-\mathscr{g}(x, t, R) \mid \\
& \leq \int_{0}^{t}|(a \otimes Q)(x, s)-(a \otimes R)(x, s)| \mathrm{d} s \\
& \leq \int_{0}^{T^{\prime}}|(a \otimes Q)(x, s)-(a \otimes R)(x, s)| \mathrm{d} s \\
& =\int_{0}^{T}|(a \otimes Q)(x, s)-(a \otimes R)(x, s)| \mathrm{d} s+\int_{T}^{T^{\prime}}|(a \otimes Q)(x, s)-(a \otimes R)(x, s)| \mathrm{d} s \\
& \leq \int_{0}^{T} \kappa \mathrm{d}_{D, 0}^{1}(Q, R) \mathrm{d} s+\int_{T}^{T^{\prime}} \kappa \mathrm{d}_{D^{\prime}, 0}^{1}(Q, R) \mathrm{d} s \\
& \leq \kappa T\|Q-R\|_{D}+\kappa\left(T^{\prime}-T\right)\|Q-R\|_{D^{\prime}} .
\end{aligned}
$$

Taking the supremum of the left-hand side over $(x, t) \in D^{\prime}$, we obtain (2.6).

To obtain (2.7), let $\delta \geq 0$ and suppose that $(x, t),\left(x^{\prime}, t^{\prime}\right) \in D$ satisfy $\left|x-x^{\prime}\right| \leq \delta$ and $\left|t-t^{\prime}\right| \leq \delta$; assume first that $t^{\prime} \geq t$. Then, applying (2.5), for cases (Bo), $\left(\mathrm{F}_{0}\right),\left(\mathrm{F}_{1}\right)$, and $\left(\mathrm{F}_{u}\right)$, we have

$$
\begin{aligned}
& \left|g\left(x^{\prime}, t^{\prime}, Q\right)-g(x, t, R)\right| \\
& \quad \leq \int_{0}^{t}\left|(a \otimes Q)\left(x^{\prime}, s\right)-(a \otimes R)(x, s)\right| \mathrm{d} s+\int_{t}^{t^{\prime}}\left|(a \otimes Q)\left(x^{\prime}, s\right)\right| \mathrm{d} s \\
& \quad \leq T\left(\kappa \mathrm{d}_{D, \delta}^{1}(Q, R)+\eta(\delta)\left(\|Q\|_{D} \vee\|R\|_{D}\right)+\beta\right)+\delta\|a\|\left(\mathrm{e}^{T}+\|Q\|_{D}\right),
\end{aligned}
$$


where $\|a\|$ is the supremum of $a$ on $[0, \infty)$. If we assume that $t^{\prime} \leq t$, we obtain a similar bound, except that the last term has $\|R\|_{D}$ in place of $\|Q\|_{D}$. Taking the above two cases into account and taking the supremum of the left-hand side, we obtain (2.7) with $\gamma=\beta+\mathrm{e}^{T}$ and $\omega(\delta)=T \eta(\delta)+\delta\|a\|$.

Definition 2.2. If $g$ satisfies the conclusions of Lemma 2.1 for some $\kappa \geq 0, \gamma \geq 0$, and $\omega(\delta) \geq 0$, then $g$ is of $\kappa$-contraction type. The operator $g$ is of contraction type if it is of $\kappa$-contraction type for some $\kappa \geq 0$.

Tautologically, under Definition 2.2, Lemma 2.1 says that if $g$ is given by (2.1) with ' $\otimes$ ' of contraction type, then $g$ is of contraction type.

Proposition 2.1. If $g_{1}$ and $g_{2}$ are of $\kappa_{1}$-contraction type and $\kappa_{2}$-contraction type, respectively, then $g_{1}+g_{2}$ is of $\left(\kappa_{1}+\kappa_{2}\right)$-contraction type, and $\alpha g_{1}$ is of $|\alpha| \kappa_{1}$-contraction type for any $\alpha \in \mathbb{R}$. Hence, the collection of contraction-type operators form a vector space over $\mathbb{R}$. The constant operator $\mathrm{g}(\cdot)=1$ is a contraction operator of type 0 .

Proof. The triangle inequality shows the first claim, the remaining claims are self evident.

We note that Proposition 2.1 shows that if $g$ is of contraction type then so is $g+1$. The next corollary shows that any $g$ of contraction type preserves continuity.

Corollary 2.1. If a $(y)$ is bounded, $Q$ is continuous on $\mathbb{R}^{+} \times \mathbb{R}^{+}$, and $q$ is of contraction type, then $g(Q)$ is continuous.

Proof. Given $\varepsilon>0$, by the uniform continuity of $Q$ in any compact domain $D$ of the form $[0, X] \times[0, T]$, and, since $\omega(\delta) \downarrow 0$ as $\delta \downarrow 0$, there exists $\delta>0$ such that $\kappa T \mathrm{~d}_{D, \delta}^{1}(Q, Q)<\varepsilon / 2$ and $\omega(\delta)\left(\|Q\|_{D}+\gamma\right)<\varepsilon / 2$. Hence, $\mathrm{d}_{D, \delta}(g(Q), g(Q))<\varepsilon$ by (2.7).

Theorem 2.1. Let $Q_{0}(x, t)$ be any continuous function on $\mathbb{R}^{+} \times \mathbb{R}^{+}$, and let $Q_{m}, m=1,2, \ldots$, be given by (2.3) for some $g$ of $\kappa$-contraction type. Then the $Q_{m}, m=0,1, \ldots$, are continuous and converge uniformly on compact subsets of $\mathbb{R}^{+} \times \mathbb{R}^{+}$to a continuous function $\bar{Q}$ that satisfies (2.4).

Additionally, for every compact domain $D \subset \mathbb{R}^{+} \times \mathbb{R}^{+}$, there exist a constant $C>0$ and $\theta \in(0,1)$ such that

$$
\left\|\bar{Q}-Q_{m}\right\|_{D} \leq C \theta^{m} \quad \text { for all } m=0,1, \ldots
$$

Proof. Let $\kappa>0$, as otherwise $Q_{m}=0$ for all $m \geq 1$ and the result is trivial. Let $X \geq 0$ be arbitrary, and let $\widetilde{T}$ be the supremum over all $T \geq 0$ such that there exist $C>0$ and $\theta \in(0,1)$ such that

$$
c_{m, T} \leq C \theta^{m} \quad \text { for } m=1,2, \ldots, \quad \text { where } \quad c_{m, T}=\left\|Q_{m}-Q_{m-1}\right\|_{[0, X] \times[0, T]} .
$$

We first prove that $\widetilde{T}=\infty$. Let $\theta \in(0,1)$. Using (2.6) with $T=0$ and $T^{\prime}=\theta / \kappa$, we obtain

$$
c_{m, T^{\prime}} \leq\left(\frac{c_{1, T^{\prime}}}{\theta}\right) \theta^{m} \quad \text { for } m=1,2, \ldots
$$

by induction, first noting that it holds trivially for $m=1$. Hence, $\widetilde{T}>0$.

If (2.8) holds for some $\mathcal{C}>0, \vartheta \in(0,1)$, and $T>0$, then, for $T^{\prime}=T+\vartheta / \kappa$, using (2.6) again,

$$
c_{m, T^{\prime}} \leq \kappa T c_{m-1, T}+\kappa\left(T^{\prime}-T\right) c_{m-1, T^{\prime}} \leq \kappa T \mathcal{C} \vartheta^{m-1}+\vartheta c_{m-1, T^{\prime}} \quad \text { for } m \geq 2 .
$$


The inequality

$$
c_{m, T^{\prime}} \leq C^{\prime} m \vartheta^{m-1}, \quad \text { where } \quad C^{\prime}=\max \left\{\kappa T \mathcal{C}, c_{1, T^{\prime}}\right\},
$$

holds trivially for $m=1$, and now induction, using (2.9), shows that it holds for all $m \geq 1$. Elementary calculus now shows that $c_{m, T^{\prime}} \leq C^{\prime} m \vartheta^{m-1}$ for $m \geq 1$ implies that, for any $\theta \in$ $(\vartheta, 1)$, there exists $C$ such that $c_{m, T^{\prime}} \leq C \theta^{m}$ for all $m \geq 1$. Hence, (2.8) holds when replacing $T$ by $T^{\prime}$, and, therefore, assuming that $\widetilde{T}<\infty$ leads to a contradiction. Now note that (2.8) holds when replacing $[0, X] \times[0, T]$ by any compact $D$, and $c_{m, T}$ by $c_{m, D}=\left\|Q_{m}-Q_{m-1}\right\|_{D}$, as $D \subset[0, X] \times[0, T]$ for some sufficiently large $X$ and $T$.

By Corollary 2.1 and induction, the functions $Q_{m}, m=0,1, \ldots$, are continuous and, by (2.8), they form a Cauchy sequence in the supremum norm on any compact domain $D$. Therefore, $Q_{m}, m=0,1, \ldots$, converges uniformly on any compact domain to a continuous limit function $\bar{Q}$. The function $\bar{Q}$ solves (2.4) as applying the triangle inequality and then (2.6) with $T^{\prime}=T$ yields

$$
\begin{aligned}
\|g(\bar{Q})-\bar{Q}\|_{D} & \leq\left\|\mathcal{g}(\bar{Q})-Q_{m+1}\right\|_{D}+\left\|Q_{m+1}-\bar{Q}\right\|_{D} \\
& =\left\|g(\bar{Q})-g\left(Q_{m}\right)\right\|_{D}+\left\|Q_{m+1}-\bar{Q}\right\|_{D} \\
& \leq \kappa T\left\|\bar{Q}-Q_{m}\right\|_{D}+\left\|Q_{m+1}-\bar{Q}\right\|_{D},
\end{aligned}
$$

whose right-hand side converges to 0 as $m \rightarrow \infty$. Hence, $g(\bar{Q})=\bar{Q}$.

Lastly, by the triangle inequality and (2.8), for all $n \geq m$,

$$
\left\|Q_{n}-Q_{m}\right\|_{D} \leq C \sum_{j=m+1}^{n} \theta^{j} \leq\left(\frac{C}{1-\theta}\right) \theta^{m+1}
$$

so letting $n \rightarrow \infty$ yields the final claim of the theorem.

Theorem 2.2. If $g$ is of contraction type, (2.4) has a unique solution that is bounded over all compact domains of $\mathbb{R}^{+} \times \mathbb{R}^{+}$.

Proof. Let $Q$ and $R$ be two solutions to (2.4) that are bounded over all compact subsets of $\mathbb{R}^{+} \times \mathbb{R}^{+}$. Let $D=[0, X] \times[0, T]$ and $D^{\prime}=[0, X] \times\left[0, T^{\prime}\right]$ for some $X \geq 0$ and $T^{\prime} \geq T \geq 0$. Taking $T=0$ and $\kappa T^{\prime}<1$ in (2.6) yields $\|Q-R\|_{D^{\prime}}=0$.

Replacing $T$ and $T^{\prime}$ by $T^{\prime}$ and $T^{\prime \prime}$, respectively, in (2.6), where $0 \leq \kappa\left(T^{\prime \prime}-T^{\prime}\right)<1$, it is easy to see that, for the larger domain $D^{\prime \prime}=[0, X] \times\left[0, T^{\prime \prime}\right]$, we now obtain

$$
\|Q-R\|_{D^{\prime \prime}}=\|\mathcal{g}(Q)-\mathcal{g}(R)\|_{D^{\prime \prime}} \leq \kappa\left(T^{\prime \prime}-T^{\prime}\right)\|Q-R\|_{D^{\prime \prime}}
$$

This implies that $\|Q-R\|_{D^{\prime \prime}}=0$, and it follows that $\|Q-R\|_{D}=0$ holds for any domain $[0, X] \times[0, T]$, and, hence, for any compact domain $D$.

In the cases of interest to us here, the operator $g$ has some additional properties, in particular, monotonicity and a type of boundedness.

Definition 2.3. An operator $g$ is $B$-bounded for a continuous function $B: \mathbb{R}^{+} \times \mathbb{R}^{+} \rightarrow \mathbb{R}^{+}$if

$$
Q \leq B \quad \text { implies that } \quad g(Q) \leq B .
$$

An operator $g$ is monotone if

$$
Q \leq R \quad \text { implies that } \quad g(Q) \leq \mathscr{g}(R) .
$$


Clearly, the operator $g$ of $(2.1)$ is monotone for cases $(\mathrm{Bo}),\left(\mathrm{F}_{0}\right),\left(\mathrm{F}_{1}\right)$, and $\left(\mathrm{F}_{u}\right)$; hence, so is $g$ of (2.2) with ' $\otimes$ ' given by case (Bo). The following proposition is immediate from the preceding definitions.

Proposition 2.2. Let $g$ be a monotone, B-bounded operator of contraction type, and let $Q_{m}, m=0,1, \ldots$, be the functions given by (2.3) when $Q_{0}(x, t) \equiv 0$, and $Q_{m}^{\prime}$ be the functions when $Q_{0}^{\prime}(x, t)=B(x, t)$. Then the unique solution $\bar{Q}$ to (2.4) satisfies

$$
0 \leq Q_{m}(x, t) \leq \bar{Q}(x, t) \leq Q_{m}^{\prime}(x, t) \leq B(x, t) \text { for all } m=0,1, \ldots
$$

If iteration (2.3) is used for numerical approximation of the solution $\bar{Q}$ to (2.4), then Proposition 2.2 allows for the verification of the precision of the calculation, as it provides lower and upper bounds for $\bar{Q}$; the distance between these bounds therefore gives the approximation error.

We now show that when $a$ is a bounded function, $g$ of (2.1) is of contraction type for cases $(\mathrm{Bo}),\left(\mathrm{F}_{0}\right),\left(\mathrm{F}_{1}\right)$, and $\left(\mathrm{F}_{u}\right)$, and that the same is true for $g$ of $(2.2)$ with ' $\otimes$ ' given in case (Bo).

Lemma 2.2. Let $a: \mathbb{R}^{+} \rightarrow \mathbb{R}^{+}$be uniformly continuous and bounded by $\kappa$. The operator $q$ given by $(2.1)$ is of $\kappa$-contraction type in cases $(B o)$ and $\left(F_{0}\right)$, of 1-contraction type in case $\left(F_{1}\right)$, and of $[(1-u) \kappa+u]$-contraction type in case $\left(F_{u}\right)$. The operator g given by $(2.2)$ with ' $\otimes$ ' as in case $(\mathrm{Bo})$ is of $\kappa$-contraction type.

Proof. For the first claim, with ' $\otimes$ ' given by case (Bo), it suffices, by Lemma 2.1, to prove that ' $\otimes$ ' is of $\kappa$-contraction type. Let a compact $D=[0, X] \times[0, T]$ and $\delta \geq 0$ be given, and let $(x, t),\left(x^{\prime}, t\right) \in D$ with $\left|x-x^{\prime}\right| \leq \delta$. For a given $\epsilon>0$, let $y^{*} \in\left[0, x^{\prime}\right]$ achieve $\sup _{0 \leq y \leq x^{\prime}} a(y) Q\left(x^{\prime}-y, t\right)$ to within $\epsilon$, and set $y_{1}^{*}=\min \left\{y^{*}, x\right\}$. Hence,

$$
\begin{aligned}
(a \otimes & Q)\left(x^{\prime}, t\right)-(a \otimes R)(x, t) \\
& \leq a\left(y^{*}\right) Q\left(x^{\prime}-y^{*}, t\right)-a\left(y_{1}^{*}\right) R\left(x-y_{1}^{*}, t\right)+\epsilon \\
& =a\left(y^{*}\right)\left[Q\left(x^{\prime}-y^{*}, t\right)-R\left(x-y_{1}^{*}, t\right)\right]+\left(a\left(y^{*}\right)-a\left(y_{1}^{*}\right)\right) R\left(x-y_{1}^{*}, t\right)+\epsilon .
\end{aligned}
$$

Since $0 \leq a(y) \leq \kappa$ for all $y \geq 0$ and $\left|\left(x^{\prime}-y^{*}\right)-\left(x-y_{1}^{*}\right)\right| \leq \delta$, with

$$
\eta(\delta)=\sup _{\left|y^{\prime}-y\right| \leq \delta}\left|a\left(y^{\prime}\right)-a(y)\right|,
$$

we obtain

$$
(a \otimes Q)\left(x^{\prime}, t\right)-(a \otimes R)(x, t) \leq \kappa \mathrm{d}_{D, \delta}^{1}(Q, R)+\eta(\delta)\|R\|_{D} .
$$

Similarly, there exist another $y^{*} \in[0, x]$ and corresponding $y_{1}^{*} \in\left[0, x^{\prime}\right]$ such that

$$
(a \otimes Q)\left(x^{\prime}, t\right)-(a \otimes R)(x, t) \geq a\left(y_{1}^{*}\right) Q\left(x^{\prime}-y_{1}^{*}, t\right)-a\left(y^{*}\right) R\left(x-y^{*}, t\right)-\epsilon,
$$

which yields a bound of the form (2.11) with the roles of $Q$ and $R$ interchanged. Since $\epsilon$ is arbitrary,

$$
\left|(a \otimes Q)\left(x^{\prime}, t\right)-(a \otimes R)(x, t)\right| \leq \kappa \mathrm{d}_{D, \delta}^{1}(Q, R)+\eta(\delta)\left(\|Q\|_{D} \vee\|R\|_{D}\right) .
$$

Taking the supremum of the left-hand side and noting that $\eta(0)=0$ and $\lim _{\delta \downarrow 0} \eta(\delta)=0$ by the uniform continuity of $a(y)$ yields the desired conclusion. The first and final claims of the lemma are now immediate. 
In case $\left(\mathrm{F}_{u}\right)$ we have, in a similar way,

$(a \otimes Q)\left(x^{\prime}, t\right)-(a \otimes R)(x, t) \leq a\left(y^{*}\right) \mathrm{e}^{t}+\left[a\left(y^{*}\right)+u\left(1-a\left(y^{*}\right)\right)\right] Q\left(x-y^{*}, t\right)-a\left(y_{1}^{*}\right) \mathrm{e}^{t}$

$$
-\left[a\left(y_{1}^{*}\right)+u\left(1-a\left(y_{1}^{*}\right)\right)\right] R\left(x-y_{1}^{*}, t\right)+\epsilon .
$$

Adding and subtracting terms as before, we obtain

$$
\begin{aligned}
& \left(a\left(y^{*}\right)-a\left(y_{1}^{*}\right)\right) \mathrm{e}^{t}+\left[(1-u) a\left(y^{*}\right)+u\right]\left[Q\left(x-y^{*}, t\right)-R\left(x-y_{1}^{*}, t\right)\right] \\
& +\left[\left(a\left(y^{*}\right)-a\left(y_{1}^{*}\right)\right)(1-u)\right] R\left(x-y_{1}^{*}, t\right)+\epsilon \\
& \quad \leq[(1-u) \kappa+u] \mathrm{d}_{D, \delta}^{1}(Q, R)+\eta(\delta) \mathrm{e}^{T}\left(\|R\|_{D}+1\right)+\epsilon,
\end{aligned}
$$

since $\left|(1-u) a\left(y^{*}\right)+u\right| \leq(1-u) \kappa+u$. The result follows as for case (Bo). Now cases $\left(\mathrm{F}_{0}\right)$ and $\left(\mathrm{F}_{1}\right)$ follow by specializing $\left(\mathrm{F}_{u}\right)$ to $u=0$ and $u=1$, respectively.

Lemma 2.3. Let $g$ be given by (2.2) for case (Bo). If $a: \mathbb{R}^{+} \rightarrow \mathbb{R}^{+}$is bounded by $\kappa$ then $g$ is $\mathrm{e}^{\kappa t}$-bounded.

Proof. If $Q(x, s) \leq \mathrm{e}^{\kappa s}$, using the fact that $0 \leq a(y) \leq \kappa$ for all $y \geq 0$, we have, for all $x \geq 0$,

$$
g(x, t, Q) \leq \kappa \int_{0}^{t} \sup _{0 \leq y \leq x} Q(y, s) \mathrm{d} s+1 \leq \kappa \int_{0}^{t} \mathrm{e}^{\kappa s} \mathrm{~d} s+1=\mathrm{e}^{\kappa t},
$$

proving the boundedness claim.

Lemma 2.4. Consider case $\left(F_{u}\right)$ with $a: \mathbb{R}^{+} \rightarrow \mathbb{R}^{+}$bounded by $\kappa$, and let $\alpha=(1-u) \kappa+u$. Then $g$ of (2.1) is $B$-bounded, where $B(x, t)=B_{\alpha}(t)$ is given by

$$
B_{\alpha}(t)= \begin{cases}\kappa t \mathrm{e}^{t}, & \alpha=1 \\ \frac{\kappa}{1-\alpha}\left(\mathrm{e}^{t}-\mathrm{e}^{\alpha t}\right), & \alpha \neq 1\end{cases}
$$

In particular, in case $\left(F_{0}\right)$ the operator $g$ is $B_{\kappa}(t)$-bounded, and in case $\left(F_{1}\right)$ it is $\kappa$ t $\mathrm{e}^{t}$-bounded.

Proof. It is easy to check in both the $\alpha=1$ and $\alpha \neq 1$ cases that if $Q(x, t) \leq B_{\alpha}(t)$ then

$$
g(x, t, Q) \leq \int_{0}^{t}\left(\kappa \mathrm{e}^{s}+\alpha B_{\alpha}(s)\right) \mathrm{d} s=B_{\alpha}(t) .
$$

The following corollary summarizes the results above.

Corollary 2.2. Let $a: \mathbb{R}^{+} \rightarrow \mathbb{R}^{+}$be uniformly continuous and bounded by $\kappa$. Then, with $\mathrm{g}$ given by (2.1) and ' $\otimes$ ' either case (Bo), $\left(F_{0}\right),\left(F_{1}\right)$, or $\left(F_{u}\right)$ or with $g$ given by (2.2) and ' $\otimes$ ' given in case (Bo), there exists a continuous solution to the equation $g(Q)=Q$, and there are no other solutions bounded over compact domains. Moreover, with $Q_{0}(x, t)$ any continuous function from $\mathbb{R}^{+} \times \mathbb{R}^{+}$to $\mathbb{R}^{+}$, the sequence of iterates of $a$ given by $Q_{m+1}=g\left(Q_{m}\right)$ for $m \geq 0$ converges uniformly to the solution of $g(Q)=Q$ at an exponential rate. For every $m$, the solution to $g(Q)=Q$ is bounded below by the $m$ th term of the sequence initialized with $Q_{0}(x, t)=0$, and if $g$ is $B$-bounded then it is also bounded above by the mth term of the sequence initialized with $Q_{0}(x, t)=B(x, t)$.

We conclude this section with a proof of Theorem 1.3. 
Proof of Theorem 1.3. Let $g$ be given by (2.1) and ' $\otimes$ ' given in case $\left(\mathrm{F}_{u}\right)$ for $u \in[0,1]$. By Lemma 2.2, when $a$ is a probability, the operator $g$ is $t \mathrm{e}^{t}$-bounded. By Corollary 2.2, the unique solution $\bar{N}$ to $g(Q)=Q$ exists, is continuous, and satisfies $0 \leq \bar{N}(x, t) \leq t \mathrm{e}^{t}$. Now letting $\bar{N}(x, t)=\mathrm{e}^{t} N(x, t)$ as in (1.6), we have $N(x, t) \in[0, t]$, as claimed.

\section{The bomber}

In this section we consider the dynamic programming equation (1.7), and specialize the results of the previous section to ' $\otimes$ ' given in case (Bo). Let $\mathcal{F}(Q)$ and $g(Q)$ be given by (2.2). In particular, (1.7) may be written more compactly as $g(\bar{P})=\bar{P}$. Throughout this section, $a(y)$ can be interpreted as the probability of the bomber surviving an enemy encounter after expending $y$ units of ammunition, although our results hold in greater generality, in particular, for any bounded log-concave $a: \mathbb{R}^{+} \rightarrow \mathbb{R}^{+}$. We require strict log-concavity for Theorem 3.2. For example, the function $a(y)$ in (1.1) is strictly log-concave in $y>0$ for any $u<1$, being the composition of a strictly concave function and a strictly increasing and concave function, the $\log$.

We show that $\mathcal{F}$ and $g$ preserve $\mathrm{TP}_{2}$ and other properties, which are used to prove Theorem 1.2, i.e. that conjecture (A) holds for the bomber problem. The fighter problems that we treat in subsequent sections have much in common with the present problem. We begin by collecting some facts about $\mathrm{TP}_{2}$ and log-concave functions in Lemma 3.1, below; the claims which cannot be easily verified using definition (1.4) can be found in Karlin (1968, pp. 16, 30, and 99). For part 1 of Lemma 3.1, see also Schoenberg (1951). Note also that part 2 is a special case of part 3, and that the second conclusion of part 5 follows from part 4 after noting that a function $f(x)$ of the single variable $x$, or $g(y)$ of $y$, is $\operatorname{TP}_{2}(x, y)$.

Lemma 3.1. 1. A nonnegative function a $(y)$ is (strictly) log-concave if and only if $a(x-y)$ is (strictly) $T P_{2}(x, y)$.

2. The convolution of log-concave functions is log-concave.

3. (The composition formula.) If $J(x, y)$ is $T P_{2}(x, y), L(y, z)$ is $T P_{2}(y, z), \sigma$ is a nonnegative $\sigma$-finite measure on $\mathbb{R}$, and $M(x, z)=\int J(x, y) L(y, z) \mathrm{d} \sigma(y)$ exists, then $M(x, z)$ is $T P_{2}(x, z)$.

4. If $J(x, y)$ and $L(x, y)$ are $\operatorname{TP}_{2}(x, y)$, then so is their product.

5. If $J(x, y)$ is $T_{2}(x, y)$ then $J(x, y)^{p}$ for any positive $p$, and $J(x, y) f(x) g(y)$ for any nonnegative functions $f$ and $g$, are $T P_{2}$.

Given a $\mathrm{TP}_{2}$ function $Q$ defined on $\mathbb{R}^{+} \times \mathbb{R}^{+}$, we extend it to $\mathbb{R} \times \mathbb{R}$ by setting it equal to 0 where it was not defined before; the resulting function is $\mathrm{TP}_{2}$ on $\mathbb{R} \times \mathbb{R}$. The next lemma follows directly from (2.1) and (2.2).

Lemma 3.2. If $a: \mathbb{R}^{+} \rightarrow \mathbb{R}^{+}$and $Q(x, t)$ is nonnegative and increasing in $x$, then $\mathcal{F}(Q)$ and $g(Q)=\mathscr{F}(Q)+1$ are nonnegative and increasing in $x$ and $t$.

The following key lemma gives conditions under which the $\mathrm{TP}_{2}$ property is preserved by the sup-convolution. We recall that ' $\otimes$ ' in this section is given by case (Bo).

Lemma 3.3. If $Q$ is a continuous, nonnegative $T P_{2}$ function on $\mathbb{R}^{+} \times \mathbb{R}^{+}$and $a: \mathbb{R}^{+} \rightarrow \mathbb{R}^{+}$ is log-concave, then $a \otimes Q$ is $T P_{2}$. 
Proof. Let $(x, t) \in \mathbb{R}^{+} \times \mathbb{R}^{+}$and $p>0$ be arbitrary. Making the change of variable $v=x-y$ and writing the limits of integration using the indicator $I(x, v)=\mathbf{1}(0 \leq v \leq x)$ we obtain

$$
\int_{0}^{x}[a(y) Q(x-y, s)]^{p} \mathrm{~d} y=\int_{-\infty}^{\infty} a(x-v)^{p} I(x, v) Q(v, s)^{p} \mathrm{~d} v .
$$

We can directly verify from (1.4) that $I(x, v)$ is $\mathrm{TP}_{2}(x, v)$. Since $a(x)$ is log-concave on $\mathbb{R}^{+}$, defining $a(x)=0$ for $x<0$ we have $a(x)$ is log-concave on all of $\mathbb{R}$, and so the product $a(x-v) I(x, v)$, and, therefore, also $a(x-v)^{p} I(x, v)$, are $\operatorname{TP}_{2}(x, v)$ on $\mathbb{R} \times \mathbb{R}$. Hence, the integrand is a product of a $\operatorname{TP}_{2}(x, v)$ function with a $\mathrm{TP}_{2}(v, s)$ function, and so the integral is $\mathrm{TP}_{2}(x, s)$ by part 3 (the composition formula) of Lemma 3.1. Raising to the power $1 / p$, we therefore have

$$
\left(\int_{0}^{x}[a(y) Q(x-y, s)]^{p} \mathrm{~d} y\right)^{1 / p} \quad \text { is } \mathrm{TP}_{2}(x, s) \text { for all } p>0 .
$$

Since $a(y)$ is log-concave on $\mathbb{R}^{+}$, it is continuous in $(0, x]$ and bounded on $[0, x]$, with a possible discontinuity at 0 , in which case there is a downward jump. Hence, for all fixed $x$ and $s$, the product $a(y) Q(x-y, s)$ has these same properties. Therefore, the limit of the $L^{p}$ norms (3.1) of the function $a(y) Q(x-y, s)$ of $y$ on $[0, x]$ converges to its essential supremum which coincides with its maximum value over $y$. Using the nonnegativity of $a(\cdot)$ and $Q(\cdot, \cdot)$, we therefore have, by the preservation of the $\mathrm{TP}_{2}$ property under limits,

$$
\lim _{p \rightarrow \infty}\left(\int_{0}^{x}[a(y) Q(x-y, s)]^{p} \mathrm{~d} y\right)^{1 / p}=(a \otimes Q)(x, s) \text { is } \operatorname{TP}_{2}(x, s) .
$$

Lemma 3.4. If $a: \mathbb{R}^{+} \rightarrow \mathbb{R}^{+}$is log-concave and $Q$ is $T P_{2}$, then $\mathcal{F}(Q)$ is $T P_{2}$.

Proof. By Lemma 3.3, $(a \otimes Q)(x, s)$ is $\mathrm{TP}_{2}(x, s)$. As $I(s, t)=\mathbf{1}(0 \leq s \leq t)$ is $\operatorname{TP}_{2}(s, t)$, the integral of the product

$$
\mathcal{F}(x, t, Q)=\int_{0}^{t}(a \otimes Q)(x, s) \mathrm{d} s=\int_{-\infty}^{\infty}(a \otimes Q)(x, s) I(s, t) \mathrm{d} s,
$$

is $\operatorname{TP}_{2}(x, t)$.

Lemma 3.5. If $Q(x, t)$ is nonnegative, increasing in $x$ and $t$, and $T P_{2}$, then so is $Q(x, t)+1$.

Proof. It is clear that $Q(x, t)+1$ is nonnegative and increasing in $x$ and $t$ whenever $Q(x, t)$ is. To demonstrate the $\mathrm{TP}_{2}$ property, we need to show that

$$
\left(Q\left(x^{\prime}, t^{\prime}\right)+1\right)(Q(x, t)+1) \geq\left(Q\left(x^{\prime}, t\right)+1\right)\left(Q\left(x, t^{\prime}\right)+1\right),
$$

where $x^{\prime}>x$ and $t^{\prime}>t$. The monotonicity and nonnegativity of $Q$ imply that the result is trivial if $Q\left(x^{\prime}, t^{\prime}\right)=0$. Otherwise, using $Q\left(x^{\prime}, t^{\prime}\right) Q(x, t) \geq Q\left(x^{\prime}, t\right) Q\left(x, t^{\prime}\right)$, we obtain

$$
\begin{aligned}
& \left(Q\left(x^{\prime}, t^{\prime}\right)+1\right)(Q(x, t)+1)-\left(Q\left(x^{\prime}, t\right)+1\right)\left(Q\left(x, t^{\prime}\right)+1\right) \\
& \quad \geq\left(Q\left(x^{\prime}, t^{\prime}\right)+1\right)\left(\frac{Q\left(x^{\prime}, t\right) Q\left(x, t^{\prime}\right)}{Q\left(x^{\prime}, t^{\prime}\right)}+1\right)-\left(Q\left(x^{\prime}, t\right)+1\right)\left(Q\left(x, t^{\prime}\right)+1\right) \\
& \quad=\frac{\left(Q\left(x^{\prime}, t^{\prime}\right)-Q\left(x^{\prime}, t\right)\right)\left(Q\left(x^{\prime}, t^{\prime}\right)-Q\left(x, t^{\prime}\right)\right)}{Q\left(x^{\prime}, t^{\prime}\right)} \\
& \quad \geq 0,
\end{aligned}
$$


where the last inequality follows since

$$
Q\left(x^{\prime}, t^{\prime}\right) \geq \max \left\{Q\left(x^{\prime}, t\right), Q\left(x, t^{\prime}\right)\right\}
$$

as $Q$ is increasing in both $x$ and $t$.

Theorem 3.1. If $a: \mathbb{R}^{+} \rightarrow \mathbb{R}^{+}$is a uniformly continuous log-concave function then the solution $\bar{P}$ to $G(Q)=Q$ is $T P_{2}$.

Proof. Let $\bar{P}_{0}=0$ and $\bar{P}_{m+1}=\mathcal{g}\left(\bar{P}_{m}\right)$ for $m=1,2, \ldots$ We first claim that the functions $\left\{\bar{P}_{m}\right\}_{m=0}^{\infty}$ are nonnegative, increasing in both $x$ and $t$, and $\mathrm{TP}_{2}$. Clearly, the claim is true for $\bar{P}_{0}$. Assume that the claim is true for $\bar{P}_{m}$ for some $m \geq 0$. By Lemma 3.2, $\mathcal{F}\left(\bar{P}_{m}\right)$ and $\bar{P}_{m+1}$ are nonnegative and increasing in $x$ and $t$, and, by Lemma 3.4, $\mathcal{F}\left(\bar{P}_{m}\right)$ is $\mathrm{TP}_{2}$. Lemma 3.5 then shows that $\bar{P}_{m+1}=\mathcal{F}\left(\bar{P}_{m}\right)+1$ is $\mathrm{TP}_{2}$, thus completing the proof of the claim. That $\bar{P}$ is $\mathrm{TP}_{2}$ follows, as $\bar{P}_{m}$ converges uniformly to $\bar{P}$ by Corollary 2.2 , and the $\mathrm{TP}_{2}$ property is preserved under (even) pointwise limits.

Proof of Theorem 1.1. By Lemmas 2.3 and 2.2, when $a$ is a probability, the operator $g$ is of 1-contraction type and $\mathrm{e}^{t}$-bounded. By Corollary 2.2, the unique solution $\bar{P}$ to $g(Q)=Q$ exists, is continuous, and satisfies $0 \leq \bar{P}(x, t) \leq \mathrm{e}^{t}$. Now recalling (1.6), i.e. that $\bar{P}(x, t)=\mathrm{e}^{t} P(x, t)$, we have $P(x, t) \in[0,1]$. It follows from Theorem 3.1 that $P$ is $\mathrm{TP}_{2}$; hence, $P$ is $\mathrm{TP}_{2}$ by part 5 of Lemma 3.1.

The next lemma modifies the result in Samuel (1970, p. 158) to continuous ammunition and time, thus allowing us to show Theorem 1.2.

Lemma 3.6. Let $R(x, z, t)$ be a positive continuous function of $x \geq z \geq 0$ and $t \geq 0$ such that the ratio $R(x, z, t) / R\left(x, z^{\prime}, t\right)$ is increasing in $t$ whenever $x \geq z^{\prime} \geq z \geq 0$, and let

$$
k(x, t)=\min \left\{y \in[0, x]: R(x, y, t)=\max _{0 \leq z \leq x} R(x, z, t)\right\} .
$$

Then $k(x, t)$ is decreasing in $t$.

Proof. For the sake of contradiction, assume that there exist $t_{1}<t_{2}$ and $z_{1}<z_{2} \leq x$ such that $k\left(x, t_{1}\right)=z_{1}<z_{2}=k\left(x, t_{2}\right)$. Then the first equality gives $R\left(x, z_{1}, t_{1}\right) / R\left(x, z_{2}, t_{1}\right) \geq 1$, as the maximum of $R\left(x, z, t_{1}\right)$ is attained at $z=z_{1}$, whereas the second equality gives $R\left(x, z_{1}, t_{2}\right) / R\left(x, z_{2}, t_{2}\right)<1$, as $k\left(x, t_{2}\right)$ is the minimum over all $y$ which attain the maximum of $R\left(x, z, t_{2}\right)$. This contradicts the assumption that $R(x, z, t) / R\left(x, z^{\prime}, t\right)$ is increasing in $t$.

Proof of Theorem 1.2. By Theorem 1.1, the solution $P(x, t)$ of (1.2) is $\mathrm{TP}_{2}$; also, from (1.2), $P(x, t) \geq \mathrm{e}^{-t}$ for all $(x, t) \in \mathbb{R}^{+} \times \mathbb{R}^{+}$. In view of definition (1.4), the ratio $P\left(x^{\prime}, t\right) / P(x, t)$ is increasing in $t$ whenever $x<x^{\prime}$, or

$$
\frac{P(x-z, t)}{P\left(x-z^{\prime}, t\right)}, \quad \text { and, therefore, } \frac{a(z) P(x-z, t)}{a\left(z^{\prime}\right) P\left(x-z^{\prime}, t\right)},
$$

is increasing in $t$ when $0 \leq z<z^{\prime} \leq x$. Applying Lemma 3.6 with the continuous function $R(x, z, t)=a(z) P(x-z, t)$ now yields the assertion that

$$
K(x, t)=\min \left\{y \in[0, x]: a(y) P(x-y, t)=\max _{0 \leq z \leq x} a(z) P(x-z, t)\right\}
$$

is decreasing in $t$, and conjecture (A) holds. 
We now consider conjecture $(\mathrm{C})$ for the bomber.

Theorem 3.2. If $a: \mathbb{R}^{+} \rightarrow \mathbb{R}^{+}$is a strictly log-concave function then conjecture $(C)$ holds for the doubly continuous bomber problem.

Proof. For $0 \leq y<x$ and $\delta=x-y$, note that conjecture (C), i.e. $x-K(x, t) \geq y-K(y, t)$, is equivalent to

$$
K(y+\delta, t) \leq K(y, t)+\delta .
$$

Inequality (3.3) holds trivially for all $y$ such that $K(y+\delta, t) \leq \delta$. To handle the case $K(y+$ $\delta, t)>\delta$, define

$$
b(x, y)=\frac{a(x)}{a(y)} \quad \text { and } \quad G_{x}(y, t)=a(y) P(x-y, t),
$$

the latter expression being the conditional probability of survival upon expending an amount $y$ of ammunition at an encounter at time $t$, when the total amount $x$ is available, and then proceeding optimally thereafter. With these definitions in place, we obtain

$$
G_{y+\delta}(v+\delta, t)=a(v+\delta) P(y-v, t)=\frac{a(v+\delta)}{a(v)} G_{y}(v, t)=b(v+\delta, v) G_{y}(v, t) .
$$

Rewriting (3.4) and using the fact that $K(x, t)$ is optimal, we have

$$
\begin{aligned}
G_{y}(v, t) & =\frac{G_{y+\delta}(v+\delta, t)}{b(v+\delta, v)} \\
& \leq \frac{G_{y+\delta}(K(y+\delta, t), t)}{b(v+\delta, v)} \\
& =\frac{G_{y+\delta}([K(y+\delta, t)-\delta]+\delta, t)}{b(v+\delta, v)} \\
& =G_{y}(K(y+\delta, t)-\delta, t) \frac{b(K(y+\delta, t), K(y+\delta, t)-\delta)}{b(v+\delta, v)},
\end{aligned}
$$

where in the third expression we used the fact that $K(y+\delta, t)>\delta$, and the last equality followed by applying (3.4) with $v=K(x, t)-\delta$.

By part 1 of Lemma 3.1, $a(y)$ is strictly $\mathrm{TP}_{2}$, which is equivalent to $b(x+\delta, x)$ being a strictly decreasing function of $x$ for $x \geq 0$. Therefore, for $v$ satisfying $v<K(y+\delta, t)-\delta$, we have

$$
\frac{b(K(y+\delta, t), K(y+\delta, t)-\delta)}{b(v+\delta, v)}<1 .
$$

Since $K(x, t)$ is the minimal amount of ammunition that can be used to proceed optimally, if the smallest maximizer of the left-hand side of (3.5) is attained at a value of $v=K(y, t)$ satisfying $v+\delta<K(y+\delta, t)$, the ratio of the $b$-functions on the right-hand side of (3.5) would be strictly less than 1 . In this case, by (3.5), $K(y+\delta, t)-\delta$ would be a better choice for $v$ than the optimal amount $K(y, t)$. Note that, by definition, $K(y+\delta, t) \leq y+\delta$, so $K(y+\delta, t)-\delta \leq y$, and, hence, is a feasible quantity to expend, given $y$ units of ammunition. Having reached a contradiction we conclude that

$$
K(y, t)+\delta \geq K(y+\delta, t),
$$

which is (3.3). 


\section{The frail fighter}

In this section we consider the frail fighter, whose goal is to shoot down as many of the enemy planes as possible. Recall that if the frail fighter does not shoot down an encountered enemy then he gets shot down with probability 1 . In this section letting ' $\otimes$ ' be given by case $\left(\mathrm{F}_{0}\right)$, the dynamic programming equation (1.5) for the frail fighter can be written as $\bar{N}(x, t)=\mathcal{g}(x, t, \bar{N})$ for $g(x, t, Q)$ given by $(2.1)$ and $\bar{N}(x, t)=\mathrm{e}^{t} N(x, t)$. Analogous to the previous section, in this and the next section $a(y)$ can be interpreted as the probability that the fighter destroys an enemy using an amount $y$ of ammunition, although our results hold for more general $a$.

The following result is parallel to Lemma 3.4.

Lemma 4.1. If $\mathrm{e}^{-t} Q(x, t)$ is nonnegative and increasing in $x$ and $t$, then so is $\mathrm{e}^{-t} g(Q)$. If $a: \mathbb{R}^{+} \rightarrow \mathbb{R}^{+}$is log-concave and $Q$ is $T P_{2}(x, t)$, then so is $g(Q)$.

Proof. It is clear that $\mathrm{e}^{-t} \mathrm{~g}(Q)$ is nonnegative and increasing in $x$ whenever $\mathrm{e}^{-t} Q$ is. Next,

$$
\begin{aligned}
\frac{\partial \mathrm{e}^{-t} g(Q)}{\partial t} & =\sup _{0 \leq y \leq x} a(y)\left[1+\mathrm{e}^{-t} Q(x-y, t)\right]-\mathrm{e}^{-t} g(Q) \\
& =f(t)-\int_{0}^{t} f(s) \mathrm{e}^{-(t-s)} \mathrm{d} s,
\end{aligned}
$$

where $f(t)=\sup _{0 \leq y \leq x} a(y)\left[1+\mathrm{e}^{-t} Q(x-y, t)\right]$. Since $\mathrm{e}^{-t} Q(x, t)$ is increasing in $t, f(t) \geq$ $f(s)$ for all $s \in[0, t]$, and

$$
f(t) \geq\left(1-\mathrm{e}^{-t}\right) f(t)=\int_{0}^{t} f(t) \mathrm{e}^{-(t-s)} \mathrm{d} s \geq \int_{0}^{t} f(s) \mathrm{e}^{-(t-s)} \mathrm{d} s .
$$

Hence, the partial derivative (4.1) is nonnegative, and $\mathrm{e}^{-t} \mathcal{G}(Q)$ is increasing in $t$.

As $Q(x, t)$ is $\mathrm{TP}_{2}$, so is $\mathrm{e}^{-t} Q(x, t)$ by Lemma 3.1. Now, by Lemma $3.5,1+\mathrm{e}^{-t} Q(x, t)$ is $\operatorname{TP}_{2}(x, t)$. As in the proof of Lemma 3.3, letting $p>0$ and $I(x, v)=\mathbf{1}(0 \leq v \leq x)$, write

$$
\int_{0}^{x} a(y)^{p}\left[1+\mathrm{e}^{-s} Q(x-y, s)\right]^{p} \mathrm{~d} y=\int_{-\infty}^{\infty} a(x-v)^{p} I(x, v)\left[1+\mathrm{e}^{-s} Q(v, s)\right]^{p} \mathrm{~d} v,
$$

which is $\mathrm{TP}_{2}(x, s)$, as in the proof of Lemma 3.3. Taking $p$ th roots and letting $p \rightarrow \infty$, we conclude that $\sup _{0 \leq y \leq x} a(y)\left[1+\mathrm{e}^{-s} Q(x-y, s)\right]$, and, therefore, also $\sup _{0 \leq y \leq x} a(y)[1+$ $\left.\mathrm{e}^{-s} Q(x-y, s)\right] \mathrm{e}^{s}$, are $\mathrm{TP}_{2}(x, s)$. Now

$\int_{0}^{t} \sup _{0 \leq y \leq x} a(y)\left[1+\mathrm{e}^{-s} Q(x-y, s)\right] \mathrm{e}^{s} \mathrm{~d} s=\int_{-\infty}^{\infty} \sup _{0 \leq y \leq x} a(y)\left[1+\mathrm{e}^{-s} Q(x-y, s)\right] I(s, t) \mathrm{e}^{s} \mathrm{~d} s$, and the final claim follows.

The proof of Theorem 4.1, which proves the first claim of Theorem 1.4, is similar to the proof of Theorem 3.1 and is thus omitted.

Theorem 4.1. If $a: \mathbb{R}^{+} \rightarrow \mathbb{R}^{+}$is a uniformly continuous log-concave function then the solution $\bar{N}(x, t)$ to $g(Q)=Q$ is $T P_{2}$, as is $N(x, t)=\mathrm{e}^{-t} \bar{N}(x, t)$.

The frail fighter's optimal policy $K(x, t)$ satisfies

$$
K(x, t)=\min \left\{y \in[0, x]: a(y)[1+N(x-y, t)]=\sup _{0 \leq z \leq x} a(z)[1+N(x-z, t)]\right\} .
$$


Applying Lemma 3.6 with $R(x, z, t)=a(z)[1+N(x-z, t)]$ we obtain conjecture (A) for the frail fighter, thus proving the last claim of Theorem 1.4.

We now prove that conjecture $(\mathrm{C})$ holds for the frail fighter.

Theorem 4.2. If $a: \mathbb{R}^{+} \rightarrow \mathbb{R}^{+}$is a strictly log-concave function then conjecture $(C)$ holds for the frail fighter.

Proof. The argument is nearly identical to the proof of Theorem 3.2, and again it suffices to only consider $y$ for which $K(y+\delta, t)>\delta$. Let

$$
b(x, y)=\frac{a(x)}{a(y)} \quad \text { and } \quad G_{x}(y, t)=a(y)[1+N(x-y, t)],
$$

the latter expression being the expected 'score' for the fighter expending $y$ units of ammunition, conditional upon an encounter at time $t$ when a total amount $x$ is available, and then proceeding optimally thereafter. With these definitions in place we obtain

$$
G_{y+\delta}(v+\delta, t)=a(v+\delta)[1+N(y-v, t)]=\frac{a(v+\delta)}{a(v)} G_{y}(v, t)=b(v+\delta, v) G_{y}(v, t) .
$$

The same lines of argument of Theorem 3.2 from (3.4) on may now be applied to yield the conclusion.

\section{The invincible fighter}

In this section we consider an invincible fighter who cannot be shot down, whose goal is to shoot down as many of the enemy planes as possible. Hence, in this section we take $g$ given by $(2.1)$ and ' $\otimes$ ' given in case $\left(\mathrm{F}_{1}\right)$, and the relevant equation may be written as $\bar{N}(x, t)=g(x, t, \bar{N})$. The role of the next lemma, and its proof, is parallel to that of Lemma 3.3.

Lemma 5.1. If $f(x)$ and $g(x)$ are log-concave functions, then so is

$$
h(x)=\sup _{0 \leq y \leq x}[f(y) g(x-y)] .
$$

If $f(x)$ and $g(x)$ are concave functions, then so is

$$
h(x)=\sup _{0 \leq y \leq x}[f(y)+g(x-y)] .
$$

Proof. By part 2 of Lemma 3.1, the convolution of log-concave functions is log-concave. Writing

$$
h(x)=\lim _{p \rightarrow \infty}\left(\int_{0}^{x}[f(y) g(x-y)]^{p} \mathrm{~d} y\right)^{1 / p}
$$

and noting that log-concavity is preserved when taking powers and under pointwise limits, we conclude that $h(x)$ is log-concave, thus proving the first claim. To prove the second claim, write

$$
h(x)=\log \sup _{0 \leq y \leq x}\left[\mathrm{e}^{f(y)} \mathrm{e}^{g(x-y)}\right]
$$

and apply the first result to the log-concave functions $\mathrm{e}^{f(y)}$ and $\mathrm{e}^{g(x)}$. 
The proof of the following theorem uses the second part of Lemma 5.1 to show that concavity is preserved under the operation in case $\left(\mathrm{F}_{1}\right)$, and then an iteration and limit argument as in the proof of Theorem 3.1. The details are omitted.

Theorem 5.1. If $a: \mathbb{R}^{+} \rightarrow \mathbb{R}^{+}$is a uniformly continuous concave function then the solution $\bar{N}(x, t)$ to the equation $g(Q)=Q$, for $g$ given in $(2.1)$ for case $\left(F_{1}\right)$, is concave in $x$ for every $t$, as is $N(x, t)=\mathrm{e}^{-t} \bar{N}(x, t)$.

The following lemma is similar to Lemma 3.6.

Lemma 5.2. If $R(x, y)$ is a continuous function and $R\left(x, y^{\prime}\right)-R(x, y)$ is increasing in $x$ for all $y^{\prime}>y$, then

$$
k(x)=\inf \left\{y: R(x, y)=\sup _{0 \leq z \leq x} R(x, z)\right\}
$$

is increasing in $x$.

Proof. To reach a contradiction, assume that there exist $x<x^{\prime}$ such that $y^{\prime}=k(x)>$ $k\left(x^{\prime}\right)=y$. Note then that by the definition of $k$, we have

$$
R\left(x, y^{\prime}\right)=R(x, k(x))>R\left(x, k\left(x^{\prime}\right)\right)=R(x, y)
$$

and

$$
R\left(x^{\prime}, y^{\prime}\right)=R\left(x^{\prime}, k(x)\right) \leq R\left(x^{\prime}, k\left(x^{\prime}\right)\right)=R\left(x^{\prime}, y\right),
$$

contradicting the assumption on $R$.

We may now provide the proof of Theorem 1.5.

Proof of Theorem 1.5. For fixed $t$, let

$$
R(x, y)=a(y)+N(x-y, t) .
$$

Note that $R\left(x, y^{\prime}\right)-R(x, y)$ being increasing in $x$ for all $y^{\prime}>y$ is equivalent to $N\left(x-y^{\prime}\right)-$ $N(x-y)$ being increasing in $x$ for all $y^{\prime}>y$, which is equivalent to $N$ being concave in $x$. Applying Theorem 5.1 and Lemma 5.2 completes the proof.

The optimal policy for the invincible fighter is given by

$$
K(x, t)=\arg \max _{0 \leq y \leq x}[a(y)+N(x-y, t)] .
$$

Proposition 5.1. If the uniformly continuous function $a: \mathbb{R}^{+} \rightarrow \mathbb{R}^{+}$is strictly concave then the optimal policy $K(x, t)$ for the invincible fighter is unique, i.e. the maximum in (5.1) is attained uniquely.

Proof. Theorem 5.1 gives the result that $N(x, t)$ is concave in $x$ for every $t$, and, hence, $N(x-y, t)$ is concave in $y$ for all fixed $x$ and $t$. Since $a(y)$ is strictly concave in $y$, so is the function $a(y)+N(x-y, t)$, and, hence, its maximum is attained uniquely.

The argument we used above to show uniqueness no longer works in general in model $\left(\mathrm{F}_{u}\right)$, in which the fighter is vulnerable to attack, where, following (1.3), the function $R(x, y)$ in the proof of Theorem 1.5 is replaced by

$$
R(x, y)=a(y)+[a(y)+u(1-a(y))] N(x-y, t) .
$$


In this case, $R\left(x, y^{\prime}\right)-R(x, y)$ being increasing in $x$ for $y<y^{\prime}$ is no longer equivalent to the concavity of $N(x, t)$ in $x$ for fixed $t$ when $u \neq 1$.

We now prove that conjecture $(\mathrm{C})$ holds for the invincible fighter.

Theorem 5.2. If the uniformly continuous function $a: \mathbb{R}^{+} \rightarrow \mathbb{R}^{+}$is strictly concave then conjecture $(C)$ holds for the invincible fighter.

Proof. The argument is similar to the proof of Theorem 3.2. As there, it suffices to only consider $y$ for which $K(y+\delta, t)>\delta$. Define

$$
b(x, y)=\exp (a(x)-a(y)) \quad \text { and } \quad G_{x}(y, t)=\exp (a(y)+N(x-y, t)),
$$

the logarithm of the latter expression being the expected 'score' for the fighter expending $y$ units of ammunition, conditional upon an encounter, at time $t$, when a total amount $x$ is available, and then proceeding optimally thereafter. With these definitions in place we obtain

$$
G_{y+\delta}(v+\delta, t)=b(v+\delta, v) G_{y}(v, t),
$$

and the lines of argument of the proof of Theorem 3.2 may now be followed to show the desired conclusion under the condition that $\exp (a(x))$ is strictly log-concave, i.e. that $a(x)$ is strictly concave.

\section{Discussion}

We have demonstrated that conjecture (A) holds for the bomber and frail fighters, (B) holds for the invincible fighter, and (C) holds for all three, so in no model have we shown that (A) and (B) hold together. In addition, we have shown uniqueness of the optimal policy only for the invincible fighter. But of all the possible conjectures which can be raised in these models, the most outstanding is that (B) holds for the bomber, and naturally one may hope to use the ideas developed in Sections 2 and 3 to settle this.

The equivalence of conjecture (B) to the log-concavity of $P(x, t)$ in $x$ was noted in Simons and Yao (1990). Hence, parallel to the proof of conjecture (A), for a given $Q(x, s)$, consider the function

$$
R(x, t)=\int_{0}^{t}(a \otimes Q)(x, s) \mathrm{d} s,
$$

where ' $\otimes$ ' is the max-convolution operator given in case (B). Using results in Prékopa (1973), one can show that $R(x, t)$ is log-concave when $a(y)$ and $Q(x, t)$ are log-concave, similarly as to how one shows that the $\mathrm{TP}_{2}$ is preserved. When $R(x, t)$ is log-concave, for any nonnegative $x_{1}, x_{2}, t_{1}$, and $t_{2}$, we have

$$
R_{12}^{2} \geq R_{1} R_{2} \quad \text { or } \quad R_{12} \geq\left(R_{1} R_{2}\right)^{1 / 2},
$$

where

$$
R_{12}=R\left(\frac{x_{1}+x_{2}}{2}, \frac{t_{1}+t_{2}}{2}\right), \quad R_{1}=R\left(x_{1}, t_{1}\right), \quad \text { and } \quad R_{2}=R\left(x_{2}, t_{2}\right) .
$$

Now to proceed as in Lemma 3.5; for $R(x, t)+1$ to be log-concave, we require that

$$
\left(R_{12}+1\right)^{2} \geq\left(R_{1}+1\right)\left(R_{2}+1\right),
$$


and, by (6.1), it suffices that

$$
2 R_{12} \geq R_{1}+R_{2} \quad \text { or } \quad R_{12} \geq \frac{1}{2}\left(R_{1}+R_{2}\right),
$$

i.e. that $R(x, t)$ is concave. However, unlike the argument in the proof of Lemma 3.5, we cannot conclude (6.2) from (6.1) even though the converse is true. The geometric mean of $R_{1}$ and $R_{2}$ in (6.1) is in general smaller than the arithmetic mean in (6.2). Moreover, numerical evidence indicates that $R(x, t)$ may fail to be concave.

Nevertheless, as the concavity of $R(x, t)$ is sufficient but not necessary for the log-concavity of $R(x, t)+1, \log$-concavity may yet be conserved in each step of the iteration, and the limiting argument pushed through as before. Taking $a(y)$ to be given in (1.1) and considering the first few iterates of (1.8), we have $\bar{P}_{0}(x, t)=0$ and $\bar{P}_{1}(x, t)=1$, which are indeed log-concave. Continuing, as $a(y)$ is increasing, we have

$$
(a \otimes 1)(x, s)=\sup _{0 \leq y \leq x} a(y)=a(x),
$$

and, therefore,

$$
\bar{P}_{2}(x, t)=\int_{0}^{t}\left(a \otimes \bar{P}_{1}\right)(x, s) \mathrm{d} s+1=\int_{0}^{t} a(x) \mathrm{d} s+1=t a(x)+1 .
$$

Since

$$
\frac{\partial^{2}}{\partial x^{2}} \log \bar{P}_{2}(x, t)=\frac{-t(1+t)(1-u) \mathrm{e}^{-x}}{(\operatorname{ta}(x)+1)^{2}} \leq 0 \quad \text { for all }(x, t) \in \mathbb{R}^{+} \times \mathbb{R}^{+},
$$

the function $\bar{P}_{2}(x, t)$ is log-concave as well. Indeed, Figure 1, in which we plot $\log \bar{P}_{m}$ for $m=1, \ldots, 10,(x, t) \in[0,10] \times\{10\}$, and $u=0$, suggests that log-concavity is preserved in these further functions as well. The figure also indicates that convergence of $\bar{P}_{m}(x, t)$ for the values considered is quite rapid, consistent with the exponential bound in Theorem 2.1. Expanding the range of $t$ back toward the origin, Figure 2 depicts $\log \bar{P}_{m}(x, t)$ for $m=1, \ldots, 7$ over the range $(x, t) \in[0,10]^{2}$, and indicates that these functions are log-concave in both variables $x$ and $t$.

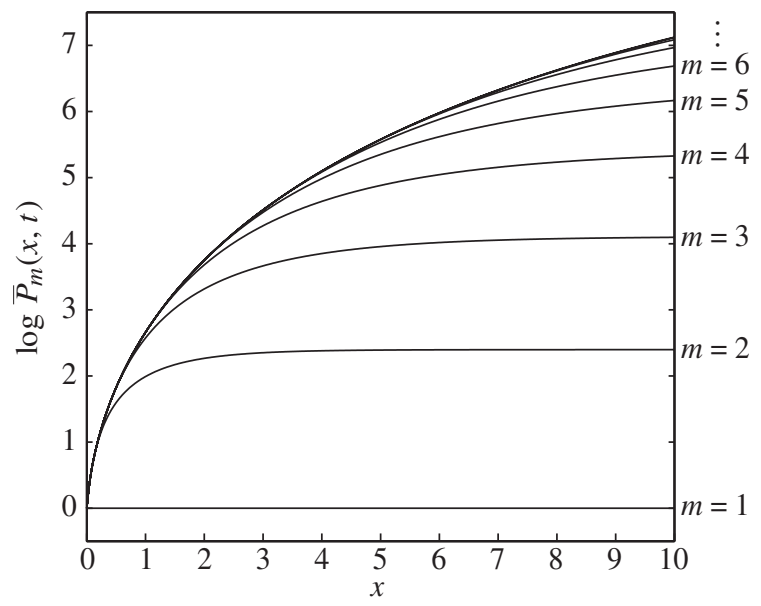

Figure 1: The functions $\log \bar{P}_{m}(x, t), m=1, \ldots, 10$, for $t=10$. 


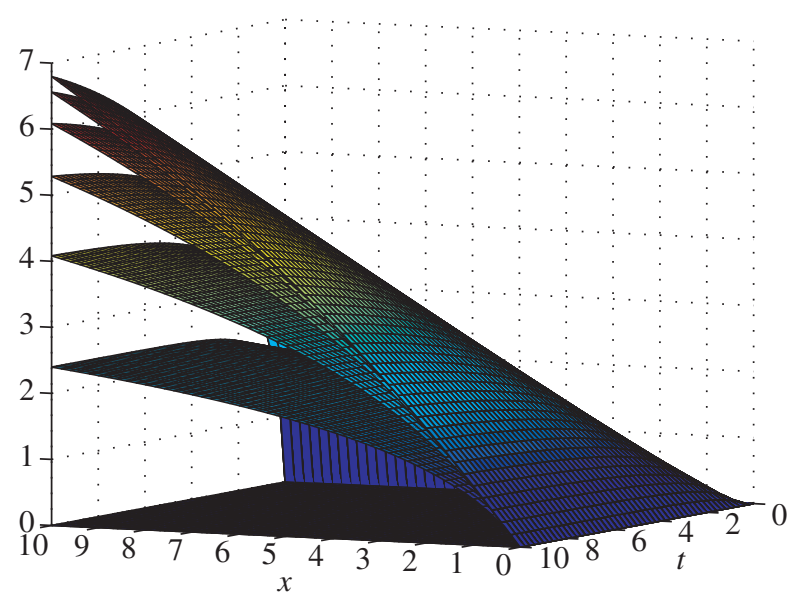

FIGURE 2: The functions $\log \bar{P}_{m}(x, t), m=1, \ldots, 7$.

\section{References}

Bartroff, J., Goldstein, L. And Samuel-Cahn, E. (2010). The spend-it-all region and small time results for the continuous bomber problem. Sequent. Anal. 29, 275-291.

Karlin, S. (1968). Total Positivity, Vol. I. Stanford University Press.

KLINGER, A. AND Brown, T. A. (1968). Allocating unreliable units to random demands. In Stochastic Optimization and Control (Proc. Adv. Seminar, Madison, Wisconsin, 1967), ed. H. F. Karreman, John Wiley, New York, pp. 173-209.

Luenberger, D. G. (1969). Optimization by Vector Space Methods. John Wiley, New York.

PRÉKOPA, A. (1973). On logarithmic concave measures and functions. Acta Sci. Math. 34, 335-343.

Samuel, E. (1970). On some problems in operations research. J. Appl. Prob. 7, 157-164.

Schoenderg, I. J. (1951). On Pólya frequency functions. I. The totally positive functions and their Laplace transforms. J. Analyse Math. 1, 331-374.

Shepp, L. A., Simons, G. and Yao, Y.-C. (1991). On a problem of ammunition rationing. Adv. Appl. Prob. 23, 624-641. Simons, G. And Yao, Y.-C. (1990). Some results on the bomber problem. Adv. Appl. Prob. 22, 412-432.

Weber, R. (1985). On a problem of ammunition rationing. In Stochastic Dynamic Optimization and Applications in Scheduling and Related Areas, Universität Passau, p. 148. 